\title{
Simulating polarized Galactic synchrotron emission at all frequencies
}

\section{The Hammurabi code}

\author{
A. Waelkens ${ }^{1}$, T. Jaffe ${ }^{2}$, M. Reinecke ${ }^{1}$, F. S. Kitaura ${ }^{1}$, and T. A. Enßlin ${ }^{1}$ \\ 1 Max Planck Institut für Astrophysik (MPA), Karl-Schwarzschildstr. 1, 85741 Garching, Germany \\ e-mail: waelkens@mpa-garching.mpg.de \\ 2 Jodrell Bank Centre for Astrophysics, University of Manchester, Alan Turing Building, Oxford Road, Manchester, M13 9PL, UK \\ Received 10 July 2008 / Accepted 15 November 2008
}

\begin{abstract}
Context. Galactic synchrotron emission, rotation measure (RM), and the deflection of ultra-high-energy-cosmic-rays (UHECR) permit detailed studies of the Galactic magnetized inter-stellar-medium (ISM). The synchrotron emission has also to be characterized properly to enable its distinction from other astrophysically interesting signals such as the CMB.

Aims. We present a publicly available code called HAMMURABI for generating mock, polarized observations of Galactic synchrotron emission with telescopes such as LOFAR, SKA, Planck, and WMAP, based on model inputs for the Galactic magnetic field (GMF), the cosmic-ray density distribution, and the thermal electron density. We also present mock UHECR deflection measure (UDM) maps based on model inputs for the GMF. In future, when UHECR sources are identified, this will allow us to use UDM as a probe of GMF in a way similar to how polarized radio sources enable us to define rotation measures.

Methods. To demonstrate the code's abilities, mock observations are compared with real data. This allows us to constrain the input parameters of our simulations with a focus on large-scale magnetic field properties.

Results. Magnetized ISM models in the literature appear to be unable to reproduce any additional observational data not included in their design.

Conclusions. As expected, attempts to model the synchrotron, UHECR-deflection, and RM input parameters indicate that any additional observational data set significantly increases the constraints on the models. The HAMMURABI code addresses this by allowing one to perform simulations of several different data sets simultaneously, providing a more reliable constraint of the magnetized ISM.
\end{abstract}

Key words. radio continuum: ISM - ISM: magnetic fields - ISM: cosmic rays

\section{Introduction}

Several different types of observation are able to probe the Galactic magnetized ISM: synchrotron radiation, rotation measure, UHECR deflection, dust-related observations (e.g. starlight polarization (Heiles 2000), and polarized dust emission), and atom-alignment spectroscopic observations, for example, have been proposed (see Yan \& Lazarian 2006).

A more robust understanding of the interstellar magnetized plasma and its radio-emission, related processes is also paramount for current and future CMB experiments (see, e.g., Miville-Deschênes et al. 2008; Page et al. 2007), where the accuracy of the measurements of the polarized CMB signal in particular will be limited by our knowledge of foreground emission (Tucci et al. 2005).

Observational studies of the magnetized ISM are usually based on a single type of observable. However, since the different observables and measurement methods provide complementary information, it is logically more sensible to constrain the magnetized ISM by considering simultaneously all possible observations. We address the need to confront those observations with models by presenting a publicly available software ${ }^{1}$

\footnotetext{
1 Software available at http://www.mpa-garching.mpg.de/ hammurabi
}

capable of generating mock synchrotron and Faraday rotation observations as well as mock UHECR deflection maps.

The code is constructed in a fashion that should allow its adaptation to complete any type of all or partial-sky line-of-sight integral observations (see, e.g., Waelkens et al. 2008; Sun et al. 2008).

We briefly summarize previous work on magnetized ISM modeling in Sect. 2, and then describe the involved Faraday rotation, UHECR deflection, and synchrotron physics, and the simplifying assumptions used in our computation in Sect. 3. The implementation of a line-of-sight (hereafter LOS) integration scheme that mimics radiative transfer is discussed in Sect. 4. We present some test output using standard, magnetic-field, thermaland cosmic-ray electron models. We discuss the role of the turbulent field and subgrid modeling in Sect. 5.1.5, and that of helical magnetic fields in Sect. 5.1.6. Finally, we present our conclusion in Sect. 6.

\section{Previous work on Galactic magnetized ISM modeling}

Several different magnetized ISM models have already been developed by the community. For example, there are the models of, Beuermann et al. (1985), Han et al. (2006), Brown et al. (2003), Haverkorn et al. (2006), Page et al. (2007), Sun et al. (2008), 
Vallée (2008), and Frick et al. (2001) for fitting parametrized Galactic models to data, and Moss \& Sokoloff (2008), Sokoloff \& Shukurov (1990), Sur et al. (2007), and Brandenburg \& Subramanian (2005) for dynamo-theory-based predictions of observational features of GMFs. Feasibility studies for radio telescopes such as LOFAR must address the modeling of smallscale GMF structures (see Jelić et al. 2008, for a first attempt). The data used are mostly total and polarized emission radio surveys of the Galaxy (Taylor et al. 2003; Gaensler et al. 2001; Haslam et al. 1982; Reich \& Reich 1986; Page et al. 2007) as well as measurements of Faraday rotation (Dineen \& Coles 2005; Johnston-Hollitt et al. 2004; Han et al. 2006) and a starlight polarization catalog compiled by Heiles (2000). Theoretical predictions are also heavily based on experience obtained from analyzing synchrotron observations of other spiral Galaxies (see e.g. Beck et al. 1996). Our work complements previous efforts by making it possible to compare simultaneously the largest possible number of observables to theoretical predictions.

\section{Physics included in the code}

We describe the physical processes and assumptions underlying our code.

\subsection{Faraday rotation}

The polarization angle of an electromagnetic wave is rotated when crossing a magnetized plasma, an effect known as Faraday rotation (see e.g. Rybicki \& Lightman 1979). The observed polarization angle $\chi$ is a function of the rotation experienced when crossing the magnetized plasma, the square of the observation wavelength, and its original (or intrinsic) angle $\chi_{0}$ at the polarized source,

$\chi=\operatorname{RM} \lambda^{2}+\chi_{0}$.

The rotation measure (hereafter RM), which quantifies the linear rate of change of the angle $\chi$ as a function of $\lambda^{2}$, is a function of the integral of the magnetic field $\boldsymbol{B}_{\mathrm{LOS}}$ along the LOS weighted by the thermal electron density $n_{\mathrm{e}}$,

$R M=a_{0} \int_{\text {here }}^{\text {there }} \mathrm{d} r n_{\mathrm{e}} B_{\mathrm{LOS}}$,

where $a_{0}=q_{\mathrm{e}}^{3} /\left(2 \pi m_{\mathrm{e}}^{2} c^{4}\right), m_{\mathrm{e}}$ is the electron mass, $c$ is the speed of light, and $q_{\mathrm{e}}$ is the electron charge.

The RM can be measured directly by a fit to Eq. (1) only in the particular case of a Faraday screen, i.e., if the observer and the polarized source have a cloud of magnetized plasma inbetween them, but no source-intrinsic, Faraday rotation occurs. In the more complicated scenario of several sources along the LOS being embedded in the magnetized plasma, the RM cannot be measured in that way since the polarization angle will no longer obey a linear dependence on $\lambda^{2}$. A complicated dependence of the polarization angles on frequency arises in such a case, a situation typically found in our Galaxy, where the synchrotron-emitting cosmic-ray electron population is embedded in the magnetized plasma that produces the Faraday rotation simultaneously.

\subsection{Synchrotron emission, total and polarized}

Relativistically moving charges in a magnetic field emit synchrotron radiation. In modeling the Galaxy we consider a cosmic-ray electron population generated mainly by supernova explosions and subsequent shock acceleration, and a GMF of strength equal to a few micro Gauss and of as a yet unknown topology. In the following, a couple of simplifying assumptions are made:

- the relativistic CR electrons have an isotropic velocity distribution, as measured to high accuracy to be the case at our location in the Galaxy (although, their propagation is anisotropic due to the presence of ordered magnetic fields; Yan \& Lazarian 2008);

- the cosmic-ray electron spectrum is assumed to be a power law with spectral index $p$. This widely used simplification is motivated by the theory of shock acceleration (a.k.a. Fermi acceleration, which predicts power-law energy distributions; Drury 1983), has been successfully applied to CMB foreground subtraction (Tegmark \& Efstathiou 1996; de Oliveira-Costa et al. 2008; Dunkley et al. 2008; Bottino et al. 2008), and is simultaneously confirmed by the measured cosmic-ray electron spectrum at Earth (e.g. Gaisser \& Stanev 2004, and references therein). However, the same observations, as well as sophisticated simulations, have shown that the power-law assumption is inadequate for the entire energy spectrum (see Strong et al. 2007). Without the powerlaw assumption and any other constraint on the shape of the energy distribution of the cosmic ray electrons implies that the analytic computations of the synchrotron emissivity are no longer possible and an integration over energy must be made, a numerically time-consuming operation that the current implementation of the code does not perform (see Sect. 5.1.3).

The synchrotron emissivity (i.e. power per unit volume per unit frequency per unit solid angle) is partially linearly polarized. Its intensity and polarization properties depend on the strength and orientation of the perpendicular (with respect to the LOS) component of the magnetic field, $B_{t}$, and the cosmic-ray electron spatial and energetic distribution. The emissivities are usually subdivided into two components, $j_{\perp, \|}=\mathrm{d} E_{\perp, \|} / \mathrm{d} t \mathrm{~d} \omega \mathrm{d} \Omega \mathrm{d} V$, respectively perpendicular and parallel to $B_{t}$, following Rybicki \& Lightman (1979) and Westfold (1959):

$$
\begin{aligned}
& j_{\perp}(\omega, \boldsymbol{r})=\frac{1}{4 \pi} \frac{\sqrt{3} q^{3}}{8 \pi m c^{2}} \omega^{\frac{1-p}{2}}\left(\frac{2 m c}{3 q}\right)^{\frac{1-p}{2}} B_{t}(\boldsymbol{r})^{\frac{p+1}{2}} C(\boldsymbol{r}) \\
& \times \Gamma\left(\frac{p}{4}-\frac{1}{12}\right)\left[\frac{2^{\frac{p+1}{2}}}{p+1} \Gamma\left(\frac{p}{4}+\frac{19}{12}\right)+2^{\frac{p-3}{2}} \Gamma\left(\frac{p}{4}+\frac{7}{12}\right)\right],
\end{aligned}
$$

and

$$
\begin{aligned}
& j_{\|}(\omega, \boldsymbol{r})=\frac{1}{4 \pi} \frac{\sqrt{3} q^{3}}{8 \pi m c^{2}} \omega^{\frac{1-p}{2}}\left(\frac{2 m c}{3 q}\right)^{\frac{1-p}{2}} B_{t}(\boldsymbol{r})^{\frac{p+1}{2}} C(\boldsymbol{r}) \\
& \times \Gamma\left(\frac{p}{4}-\frac{1}{12}\right)\left[\frac{2^{\frac{p+1}{2}}}{p+1} \Gamma\left(\frac{p}{4}+\frac{19}{12}\right)-2^{\frac{p-3}{2}} \Gamma\left(\frac{p}{4}+\frac{7}{12}\right)\right] .
\end{aligned}
$$

Here $C$ depends on the position in the Galaxy and is defined by $N(\gamma) \mathrm{d} \gamma=C \gamma^{-p} d \gamma, \gamma$ being the Lorenz factor, $N(\gamma)$ the number density of electrons $\in[\gamma, \gamma+d \gamma]$, and $p$ the spectral index as mentioned above. The charge of the electron is given by $q_{\mathrm{e}}$, the mass by $m_{\mathrm{e}}$, and $\omega=2 \pi v$, where $v$ is the observation frequency. The specific intensity $I$ is a function of observation frequency and LOS direction $\hat{\boldsymbol{n}}$ given by

$I(\omega, \hat{\boldsymbol{n}})=\int_{0}^{\infty} \mathrm{d} r\left[j_{\perp}(\omega, r \hat{\boldsymbol{n}})+j_{\|}(\omega, r \hat{\boldsymbol{n}}]\right.$, 
and the polarized specific intensity $P$ expressed as a complex variable is (see Burn 1966):

$P(\omega, \hat{\boldsymbol{n}})=\int_{0}^{\infty} \mathrm{d} r\left(j_{\perp}(\omega, r \hat{\boldsymbol{n}})-j_{\|}(\omega, r \hat{\boldsymbol{n}})\right) \mathrm{e}^{-2 i \chi(r \hat{\boldsymbol{n}})}$.

The intrinsic emission polarization angle $\chi_{0}$ is given by the inclination towards the Galactic North Pole of the local perpendicular-to-the-LOS component of the magnetic field at each position in space (same convention as adopted in Page et al. 2007). The Stokes I, Q, and U parameters ${ }^{2}$ are then represented by integrals over solid angle $\Omega$ :

$I=\int \mathrm{d} \Omega I$

and

$Q+i \mathrm{U}=\int \mathrm{d} \Omega P$

\subsection{Free-free scattering}

Free-free emission, also referred to as bremsstrahlung, arises from the scattering of charged particles by each other. Following Rohlfs \& Wilson (1996), we write the free-free intensity (in Kelvin) as

$I_{\mathrm{ff}}(\omega)=\int_{0}^{\tau_{\max }} \mathrm{d} \tau T_{\mathrm{e}}(\tau) \mathrm{e}^{-\tau(\boldsymbol{r}, \omega)}$,

where $I_{\mathrm{ff}}$ is the measured temperature (in Kelvin), $T_{\mathrm{e}}$ is the electron temperature (in Kelvin), and the optical depth is given by

$\tau=8.235 \times 10^{-2} T_{\mathrm{e}}^{-1.35}(\boldsymbol{r})(\omega / 2 \pi)^{-2.1} \operatorname{EM}(\boldsymbol{r})$.

Here the frequency $\omega / 2 \pi$ is in $\mathrm{GHz}$ and the emission measure (EM) (in pc/ $\mathrm{cm}^{-6}$ ) is

$E M=\int_{r_{0}}^{r_{1}} \mathrm{~d} r n_{\mathrm{e}}^{2}$

Furthermore, at low frequencies free-free absorption is also significant for synchrotron radiation, which is attenuated by a factor $\exp (-\tau)$.

\subsection{UHECR propagation}

UHECR's are deflected by the GMF due to the Lorentz force. The Larmour radius for relativistic particles is $r_{q}=p c / Z q_{\mathrm{e}} B_{t}$, $Z q_{\mathrm{e}}$ being the charge of the UHECR, and $p$ the momentum perpendicular to the magnetic field $B_{t}$. Provided the sources of UHECRs can be identified, an UHECR deflection measure (UDM) can be extracted from the UHECR arrival distribution by fitting the arrival data (position and energy). The net deflection of an UHECR can be approximated by the LOS integral (see e.g. Kachelrieß et al. 2007)

$\Theta_{\mathrm{offset}} \approx \int \mathrm{d} l \boldsymbol{r}_{g}^{-1}=\frac{Z q_{\mathrm{e}}}{\mathrm{pc}} \int \mathrm{d} l \boldsymbol{B}_{t} \equiv \frac{Z q_{\mathrm{e}}}{\mathrm{pc}} \boldsymbol{U} \boldsymbol{D} \boldsymbol{M}$.

${ }^{2}$ Here the specific intensities are written in italics $(I, Q, U)$, while the observed Stokes parameters (I, Q, U) are denoted by Roman letters.

\section{Implementation}

We present the implementation of the physics described in Sect. 3 and the technical characteristics of the HAMMURABI code. Given a

- 3D GMF model,

- 3D cosmic-ray electron density model;

- 3D thermal electron density model;

HAMMURABI computes full sky maps for

- the Galactic RM contribution to the extra-galactic sources;

- synchrotron I, Q, and U Stokes parameters, taking into account intrinsic Galactic Faraday depolarization;

- free-free emission and;

- UHECR deflection intensity and orientation maps.

The sky maps in HAMMURABI are subdivided into equal area pixels following the HEALPix ${ }^{3}$ pixelization scheme of Górski et al. (2005). The total number of pixels for an all-sky map, which defines the angular resolution, is $N_{\text {pix }}=12 \mathrm{NSIDE}^{2}$, with NSIDE $=2^{k}$ and $k=0, \ldots, 13$ (a HEALPix package limitation, which can be altered, see Appendix A). The angular size of a pixel $(\Delta \theta)$ can be approximated by

$\Delta \theta \approx \sqrt{\frac{3}{\pi}} \frac{3600^{\prime}}{\mathrm{NSIDE}}$.

The observation volume associated with one of the HEALPixmap pixels is sampled along the LOS at a constant radial interval $\Delta r$. As a consequence of the conical shape of the implied effective observation beam, the volume units increase with radius, and hence the weights of the sampling points along the LOS also increase. To limit the amount of non-homogeneity in the sampling, the code allows the volume resolution to be increased by splitting the beam into subbeams at some radius, which subsequently can be split further later on. We refer to the the implied 3-D sampling grid as the "3D HEALPix grid" (for further details, see Appendix A).

Formally, the maximally achievable volume resolution, i.e. the largest volume unit at the finest possible resolution $\Delta \theta \sim$ $0.43^{\prime}$, (corresponding to NSIDE $=2^{13}$ ) is given by $V_{\text {ext }}=$ $4 \pi R_{\max }^{2} \Delta r\left(12 \cdot 2^{26}\right)^{-1} \sim(4 \mathrm{pc})^{3}$ for $R_{\max } \sim 32 \mathrm{kpc}$ and $\Delta r=4 \mathrm{pc}$, since we impose an approximately cubic volume unit according to $\Delta r \sim\left(4 \pi R_{\max }^{2} / 12 \cdot 2^{26}\right)^{1 / 2}$. This implies that variations in the input parameters (e.g. magnetic field and thermal electron distribution) on scales smaller than this volume can only be taken into account with subgrid modeling if they persist to the largest radii simulated. We however, note that this is an upper limit because fluctuations are probably stronger closer to the Galactic disk, where resolution, due to the cone-like shape of the observational volume, is in all cases higher.

The quantities described in Sect. 3 are obtained by means of a discretization of the respective integrals, as detailed in Appendix A. We note that Sun et al. (2008) enhanced the original code by introducing free-free absorption. This implementation is relevant mainly to frequencies below $408 \mathrm{MHz}$. Furthermore, Sun et al. (2008) also introduced an empirical coupling between the thermal electron density and the random component of the $\mathrm{RM}$. This implementation is necessary to explain the degree of depolarization in their simulated $1.4 \mathrm{GHz}$ map. Although present in the code, it is not described here (for further details, see Sun et al. 2008).

\footnotetext{
${ }^{3}$ http://healpix.jpl.nasa.gov
} 
The 3D HEALPix grid, since the value of one observation pixel stems from the contribution of several subbeams, allows one to take into account approximately effects such as beam depolarization (see Appendix A.1 for details).

\subsection{Features}

HAMMURABI is also suitable for simulations of partial sky coverage. A single pixel or a list of pixels, representing either patches or separate locations on the sky output maps, can be computed. There is also the option to compute RMs for an individual pixel only out to a specified distance. This is relevant to simulating $\mathrm{RM}$ observations of radio pulsars or other polarized sources with distance information, in our own Galaxy. Polarized sources along the same LOS allow fractions of the ISM plasma to be probed, unlike extragalactic polarized sources, which enable the study of the integrated RM along the entire LOS through the Galaxy. The mock observations in this case are completed without beam-splitting and at the highest possible angular resolution, since RM are effectively obtained from point sources.

\section{Examples}

We select standard 3D input models to demonstrate the capabilities of the code. It is not the scope of this work to present new scientific findings, but to present a proof of concept of all that can be achieved by HAMMURABI. An application of the code was presented by Sun et al. (2008), and we briefly refer to their results here. Their preferred GMF model and cosmic-ray electron model, derived by attempting to fit a broad range of observables, are compared to the corresponding models suggested by Page et al. (2007), and fitted to reproduce solely the polarization angles observed by the WMAP satellite. Sun et al. (2008) demonstrated that the Page et al. (2007) model and every model in the literature analyzed in their work were unable to reproduce different sorts of observations of the magnetized ISM satisfactorily, since they are all constructed by considering only a particular sort of data. The discrepancies of the models presented here, visible by eye, illustrate the code's abilities.

\subsection{Inputs}

All input models presented here are optional and can be easily substituted by other models.

\subsubsection{The thermal electron density model}

For the thermal electron distribution, we use the NE2001 model (see Cordes \& Lazio 2002, 2003). This model subdivides the Galaxy into several large-scale structure elements such as a thin disk, a thick disk, spiral arms, as well as some local smallscale elements such as supernova bubbles. The NE2001 model presents a smooth thermal electron distribution. To take into account the clumpiness of the medium, we introduce a filling factor $f_{\mathrm{e}}(z)=0.07 \exp (2|z|)$, (see Berkhuijsen et al. 2006; Sun et al. 2008 , for details) where $z$ is the distance from the Galactic plane in $\mathrm{kpc}$.

\subsubsection{Thermal electron temperature model}

Sun et al. (2008) proposed a thermal electron temperature $\left(T_{\mathrm{e}}\right)$ model

$T_{\mathrm{e}}(\boldsymbol{r})=5780+287 r-526|z|+1770 z^{2}$, based on work by Quireza et al. (2006), Reynolds et al. (1999), and Peterson \& Webber (2002), where $T_{\mathrm{e}}$ is in Kelvin and the length scales are in $\mathrm{kpc}$.

\subsubsection{The cosmic-ray electron density model}

We use the cosmic-ray electron density models suggested by Page et al. (2007) and Sun et al. (2008).

- The model in Page et al. (2007) consists of an exponentially decaying disk with characteristic height $h_{d}=1 \mathrm{kpc}$ and characteristic radius of $h_{r}=5 \mathrm{kpc}$. We note that to compute the synchrotron emissivity, as can be seen in Eqs. (3) and (4), we require knowledge of the spatially dependent function $C$, not the cosmic-ray electron density. However, since we are assuming a Galaxy-wide, unique, power-law, energy spectral slope with a spectral index $p=3$ (note that in principle the code allows us to associate a different spectral index with each volume unit), these quantities are proportional to each other. The value of $C_{\text {Earth }}=6.4 \times 10^{-5} \mathrm{~cm}^{-3}$ at Earth's position is observed (see e.g. Fig. 4 of Strong et al. 2007), and although it is unclear that this is representative of other regions in the Galaxy (Sun et al. 2008; Pohl \& Esposito 1998; Strong et al. 2004), we use it as a zeroth order approximation of the normalization (which is neither necessary nor provided by Page et al. 2007) of our distribution,

$C=C_{0} \exp \left[-r / h_{r}\right] \operatorname{sech}^{2}\left(z / h_{d}\right)$,

where $r$ is the Galactic radius, while $z$ is the height, and $C_{0}$ is such that $C=C_{\text {Earth }}$ at Earth's position.

- Sun et al. (2008) proposed

$C(R, z)=C_{0} \exp \left(-\frac{R-R_{\odot}}{8 \mathrm{kpc}}-\frac{|z|}{1 \mathrm{kpc}}\right)$

where $C_{0}=C_{\text {Earth, }}$ while $C(R<3 \mathrm{kpc})=C(R=3 \mathrm{kpc})$ and $C(|z|>1)=0$. The abrupt truncation at a scale height of $|z|>1$ is necessary to accommodate low synchrotron emission at high latitudes, where an anomalously strong halo magnetic field is required to account for high RM measurements. Sun et al. (2008) cautioned that this appears unrealistic and suggests that a larger scale height of the thermal electron density (roughly twice as large as the one suggested by Cordes \& Lazio 2002) could resolve the problem by diminishing the required value of the halo magnetic field. This was confirmed by a joint analysis of pulsar dispersion measures and $\mathrm{H} \alpha$ emission by Gaensler et al. (2008). Furthermore the spectral index $p$ is 3 for observational frequencies higher than $408 \mathrm{MHz}$ and 2 otherwise. Sun et al. (2008) adopted this simplification based on observations of Roger et al. (1999) and Reich \& Reich (1988a,b), which support a flatter spectrum below $408 \mathrm{MHz}$.

The frequency dependence of the observed spectral index, which is ajusted by hand in the Sun et al. (2008) model, occurs, we emphasize, because the assumption of a single power-law energy distribution of the cosmic-ray electrons (used in this work) is not applicable to the entire spectrum (as mentioned in Sect. 3.2). To reproduce the frequency dependence of the observed spectral index in a consistent way, an extension of the code is planned, incorporating outputs of simulations such as GALPROP (Strong et al. 2007), which present spatially varying density and nonpower-law like cosmic-ray energy spectra, into HAMMURABI. We note that this also implies that an integration over all energies would be required, significantly increasing the computation time. 


\subsubsection{Large-scale Galactic magnetic-field models}

The behavior of the synchrotron emissivity is dependent mainly on the magnetic-field distribution, as shown in Eqs. (3) and (4).

It is a common practice to subdivide the Galactic magnetic field into large-scale and small-scale components. The latter will be addressed in Sect. 5.1.5. The subdivision into these two classes of fields is somewhat arbitrary, and we adopt the convention that the large-scale field is statistically anisotropic at any point in the Galaxy, while the small-scale field is not.

GMF modeling has been completed in direct studies of the Galactic magnetic field. See Page et al. (2007), Sun et al. (2008), Jansson et al. (2007), Haverkorn et al. (2006), Brown et al. (2003), Han et al. (2006), and others; in the context of UHECR propagation, see Kachelrieß et al. (2007), Tinyakov \& Tkachev (2002), Prouza \& Šmída (2003), and Harari et al. (2000). Inspired by observations of the radio-polarization patterns of other spiral galaxies, GMF models typically, present spiral-like structures. We present simulations using the model of Page et al. (2007) and the favored (ASS + RING) model proposed by Sun et al. (2008). The expressions are reproduced in Appendix B. Alternative magnetic field models could easily be incorporated into the code.

\subsubsection{Small-scale magnetic field}

Although the turbulent component of the GMF has been studied extensively (see e.g. Haverkorn et al. 2003; Han et al. 2004; and Haverkorn et al. 2006, 2008), there is, to our knowledge, not yet any model that describes it to a satisfactory degree. Observational constraints on its strength relative to that of the large-scale field imply that it is of roughly the same amplitude (i.e. a couple of micro Gauss, Beck 2001). Given a magneticfield power spectrum, HAMMURABI allows one to simulate, a Gaussian random-field realization. A realistic turbulent field, however, need not be Gaussian, since simulations of turbulence contain sheet-like structures that evidently differ from that expected of a Gaussian field (see e.g. Schekochihin et al. 2004; and Schekochihin \& Cowley 2006). This implies that the Gaussian random field should by construction reproduce the correct two point statistics of a true field, although it will not reproduce the higher order statistics because real fields are not observed to be Gaussian (see e.g. the figures in Jaegers 1987; and Clarke \& Ensslin 2006). Furthermore, this modeling assumes that the power spectrum of the field is known, which in the case of the Galaxy, to our knowledge, has not yet been determined. There are, however, measurements of the magnetic power spectrum in the intra-cluster medium (e.g. Vogt \& Enßlin 2005; Guidetti et al. 2008; Govoni et al. 2006). Furthermore, we note that the random field simulation is performed on a Cartesian grid and then interpolated to the 3D HEALPix grid, from which the mock observations are computed. This interpolation can introduce an error, which is small if the 3D HEALPix grid has volume units at least as small as those of the Cartesian grid. We also note that the Gaussian random field is not divergence-free, although we refer the reader to Sect. 5.1.6.

\subsubsection{Helical fields}

Helical magnetic fields have a broken mirror symmetry and can be illustrated most effectively by twisted flux tubes. Topological helicity is given by $\int \mathrm{d} V A \cdot B$, where $A$ is the vector potential of the field, and $B=\nabla \times A$. Current helicity is given by $\int \mathrm{d} V j \cdot B$, where $j$ is the electrical current, satisfying $j=\nabla \times B$. Both types of helicity are closely related. Topological helicity is an almsot conserved quantity, even in non-ideal MHD, and plays a crucial role in magnetic-dynamo theory. Therefore, HAMMURABI, in addition to its own in-built, power-spectrum-based, Gaussianrandom-field generator, was adapted to accept random smallscale fields with magnetic helicity generated by a separate code. This code, also available for download, is called GARFIELDS (first applied in Kitaura \& Enßlin 2008). The field becomes divergence-free in Fourier space by subtracting the component parallel to the wave vector and multiplying it by $3 / 2$ to compensate for the loss of power (which is valid in the case of isotropy)

$\hat{B}_{i}^{\prime}=\frac{3}{2}{\hat{B^{\prime \prime}}}_{i}\left(1-\bar{k}_{i}\right)$

where $\hat{B}^{\prime \prime}$ is the original Gaussian random field, while the divergence-free component $\hat{B}_{i}^{\prime}=\hat{B}_{i}^{\prime}(\boldsymbol{k})$ is such that $\left\langle\hat{B}_{i}^{\prime} \hat{B}_{j}^{\prime *}\right\rangle=$ $\left(\delta_{i j}-\bar{k}_{i} \bar{k}_{j}\right) P(k) / 2$, where $P(k)$ is the magnetic power spectrum (for details see Robertson 1940). The hat denotes a Fourier transform, $\delta$ is the Kronecker delta, $\bar{k}_{i}$ is an unit vector, and $\epsilon_{i j k}$ is the permutation symbol. The helicity is imprinted onto the divergence free Gaussian random field $\boldsymbol{B}^{\prime}$ by

$\hat{B}_{m}=\frac{1}{\sqrt{1+\alpha^{2}}}\left(\delta_{j m}+i \alpha \epsilon_{i j m} \bar{k}_{i}\right) \hat{B}_{j}^{\prime}$,

where Maximal helicity is found for $\alpha=-1$ and 1 , and leads to a magnetic correlation tensor of the form

$\left\langle\hat{B}_{i} \hat{B}_{j}^{*}\right\rangle=\left[\frac{1}{2}\left(\delta_{i j}-\bar{k}_{i} \bar{k}_{j}\right)-i \epsilon_{i j k} \bar{k}_{k} \frac{\alpha}{1+\alpha^{2}}\right] P(k)$.

We note that the divergence-free and helical properties of the simulated small-scale field only hold true in the Cartesian grid onto which GARFIELDS writes the field. As above, when projecting this onto the 3D HEALPix grid, an error occurs, which is smaller for finer 3D HEALPix grids. Furthermore, we note that the separately specified large-scale field does not need to posses any of the aforementioned characteristics.

\subsection{Synchrotron and RM outputs: comparison with observations}

We compare our example simulation with observed data. The mock total intensity $I$, polarized intensity $P$, polarization angle PA and RM maps for our two different field configurations, and slightly different cosmic-ray electron distributions of Sun et al. (2008) and Page et al. (2007) are shown in comparison to the observed synchrotron emission at $0.408 \mathrm{MHz}$ (total intensity) and $23 \mathrm{GHz}$ (polarized emission), and a RM map extrapolated from the existing still sparse RM observations (Fig. 1). All maps in this figure and throughout the paper are presented at a resolution of NSIDE $=128, \Delta r=0.21 \mathrm{kpc}, H_{\mathrm{obs}}=2, H_{\max }=3$ (see definitions in Appendix A). A Gaussian random field with a Kolmogorov spectrum $k^{-5 / 3}$ and a lower cutoff at $k_{0}=1 \mathrm{kpc}^{-1}$ (see, e.g., Han et al. 2004), and an upper cutoff given by the Nyquist frequency of the simulation box $k_{\text {Nyquist }}=3.2 \mathrm{kpc}^{-1}$, was added to both large-scale GMF models. We caution that this scale range is insufficient to describe the turbulent field in the Galaxy, as is evident from the even smaller-scale fluctuations present in the observations, although it serves our demonstration purposes. Following Sun et al. (2008), the random field amplitude is $\left\langle B_{\mathrm{ran}}^{2}\right\rangle^{1 / 2}=3 \mu \mathrm{G}$. 


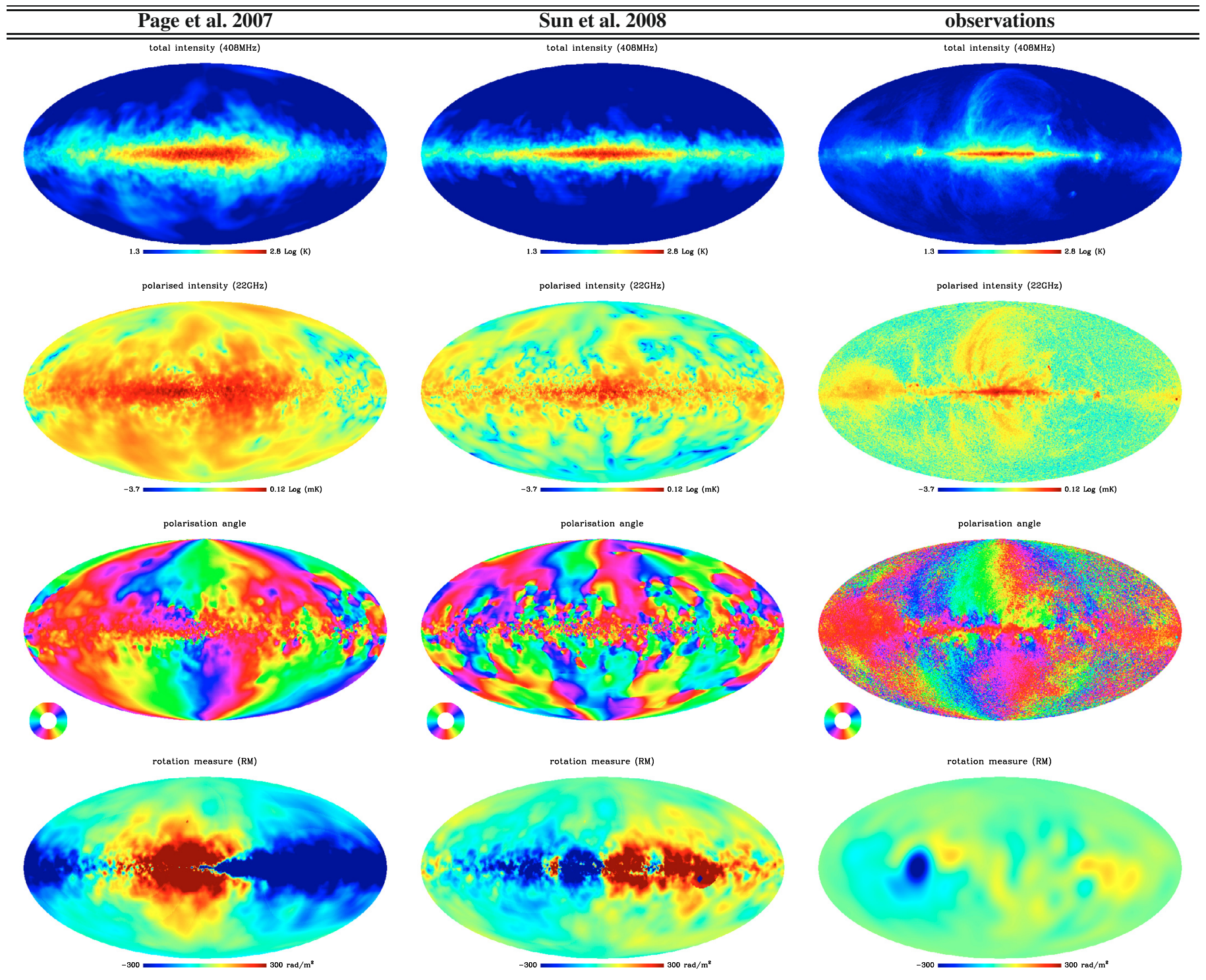

Fig. 1. Comparison of mock observations based on models from Page et al. (2007) and Sun et al. (2008) with observed data. Each row corresponds to total intensity $I$, polarized intensity $P$, polarization angle PA and rotation measure RM respectively. The third column contains the observables. $I$ is from Haslam et al. (1981, 1982), $P$ and PA are WMAP observations (Page et al. 2007; Hinshaw et al. 2008), while RM is from Dineen \& Coles (2005) derived from the Simard-Normandin et al. (1981) data.

Magnetized ISM models in the literature are designed mostly to fit a selected set of data. For example, Page et al. (2007) reproduced PA observations, and, as can be seen in Fig. 1, were abel to do this remarkably well. However, their model was evidently unable to reproduce any of the other observations (as shown by Sun et al. 2008). Furthermore, Sun et al. (2008) noted that most spiral magnetic-field configurations have similar PAs, suggesting a highly degenerate fitting problem.

The Sun et al. (2008) models for the GMF and cosmic-ray electron distributions were designed to reproduce longitude and latitude RM profile observations and $I$ and $P$ observations at $408 \mathrm{MHz}, 1.4 \mathrm{GHz}$, and $22.8 \mathrm{GHz}$, but no optimization was performed for the PA observations. We note also that Sun et al. (2008), unlike Page et al. (2007) who completed a parameter scan, adjusted the model parameters based on trial and error. The attentive reader might notice a faint horizontal-stripe-like pattern in the $I$ and $P$ simulations of this model, which is due to a combination of our limited radial step size $\Delta r=0.21 \mathrm{kpc}$, and an abrupt cut in the cosmic-ray electron density at $1 \mathrm{kpc}$ away from the Galactic plane (as discussed in Sect. 5.1.3). In other words, the code cannot resolve a sharp cut. A smaller $\Delta r$, of course, would cause the feature to vanish, although it would be inconsistent with our recommendation in Appendix A to define $\Delta r$ such that the maximum volume unit at the largest shell is close to a cubic form. The most striking difference between the two models lies probably in the different RM maps, mainly due to so-called field reversals in the Sun et al. (2008) model, which are absent in the WMAP model.

\subsection{Helicity in the ISM?}

Our code provides the possibility to study the effects of magnetic helicity on observations. In Fig. 2, field realizations with and without helicity are compared. Although they are clearly different, there is no qualitative difference between the single frequency maps from fields with helicity and without. This 


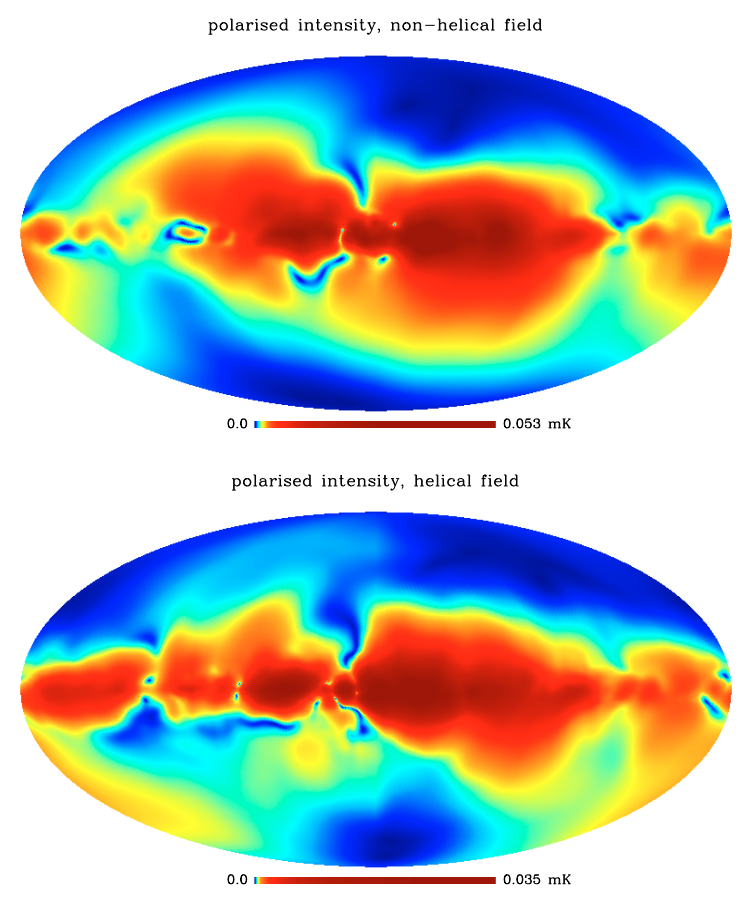

Fig. 2. Polarized intensity of a field without and one with helicity. The colors in the plot are equally distributed over an area to enhance the visualization of structures at the expense of proportionality information.

implies that measuring helicity in the ISM is difficult and the necessary multi-frequency methods required for its measurement in Faraday tomography observations (Brentjens \& de Bruyn 2005; Schnitzeler et al. 2007) still have to be developed. The availability of a tool for generating mock observations might be of assistance in developing these methods and feasibility studies for observations designed to determine the helicity in the ISM.

\subsection{UHECR deflection outputs}

As a generic tool for studying the GMF, HAMMURABI also helps in preparing UHECR-based magnetic field studies, once the sources of the UHECR particles have been identified. We included the option to compute the deflection of ultra-relativistic charges by the GMF in the code. This is yet another observable by which the GMF may be further constrained in the future. Presently, there are only a couple of hundred registered events and their origin is still speculative (The Pierre Auger Collaboration et al. 2007). Figure 3 represents the deflection intensity for the large-scale Sun et al. (2008) model. We note that the strong halo field is controversial (as discussed in Sect. 5.1.3). The deflection angle of an individual UHECR is calculated by multiplying the deflection intensity map by $Z q_{\mathrm{e}} / p c$ for this particle, as in Eq. (9).

\subsubsection{A LOFAR, SKA, WMAP, Planck tool}

The frequency range of HAMMURABI synchrotron simulations, from $\sim 100 \mathrm{MHz}$ to $\sim 100 \mathrm{GHz}$, covers currently running experiments such as WMAP as well as the upcoming generation of radio telescopes such as Planck, LOFAR and SKA. HAMMURABI has been developed to support the scientific exploitation of the data of these experiments and to provide synthetic observations for design studies. It is capable of generating full- or partial-sky

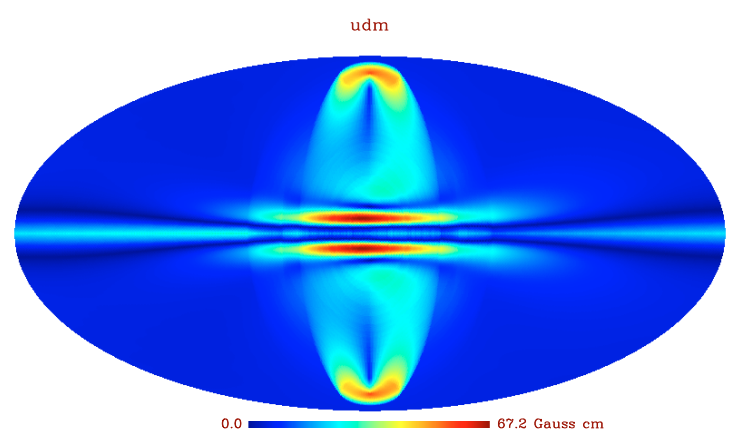

Fig. 3. UHECR deflection measure map from the Sun et al. (2008) largescale magnetic field model. To date, only a couple of hundred UHECR detections have been reported.
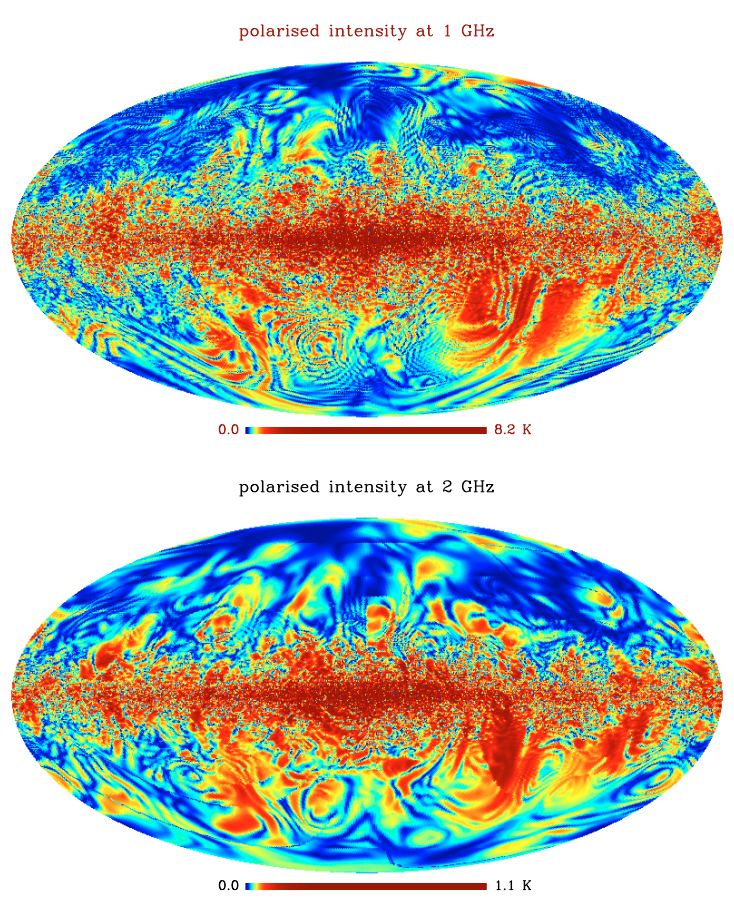

Fig. 4. Simulated polarized intensity $P$ at 1 and at $2 \mathrm{GHz}$ of the Sun et al. (2008) model. The colors in the plot are equally distributed over area to enhance the visualization of structures at the expense of proportionality information. The Faraday depolarization effect changes the complex patterns as a function of the wavelength. The higher thermal electron density on the Galactic plane keeps Faraday depolarization active long after it already ceased to be relevant in the Galactic halo.

(not shown) maps as well as individual LOS (see Fig. 5), which are useful for non-diffuse measurements, such as RMs.

Figure 4 shows the full-sky polarized intensity $\mathrm{P}$ for the aforementioned Sun et al. (2008) model (applying their low frequency corrections due to free-free absorption and a random RM enhancement factor, motivated by a coupling between thermal electrons and the random magnetic field). This model was shown to reproduce the global features of latitude and longitude profiles of observations at $1.4 \mathrm{GHz}$. Our intention was to demonstrate the effect of Faraday depolarization, which, as can be seen, changes the patterns on the maps as a function of frequency.

In principle, multi-frequency simulations permit one to take into account beam-width depolarization effects. Since measurements completed using true telescopes correspond to an integral over some frequency band, bandwidth depolarization will 


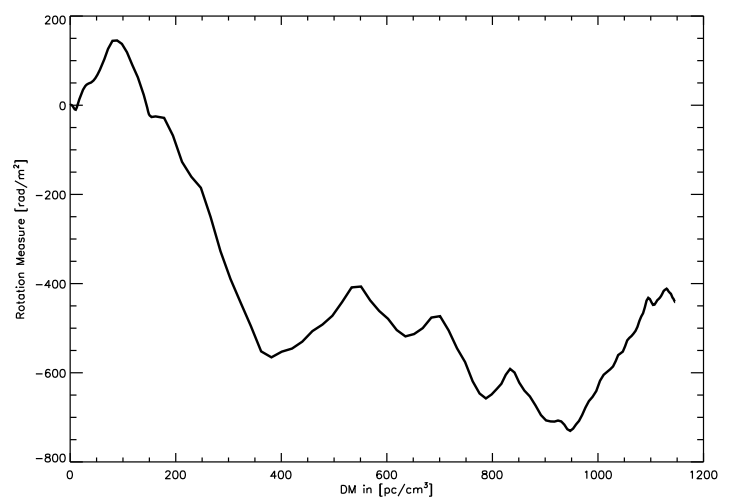

Fig. 5. The simulated rotation measure as a function of dispersion measure. We used a Gaussian random field with an upper cutoff of $5 \mathrm{kpc}$ and a root-mean-square of $6 \mu$ Gauss. Pulsar polarization measurements are, with the advent of SKA, expected to become numerous, allowing us to chart the GMF in three-dimensions, as shown here, where the RM is plotted as a function of the DM for one LOS.

also occur provided the Faraday effects are sufficiently strong across the bandwidth. Although bandwidth depolarization is not a standard HAMMURABI feature, it can be emulated by coadding closely spaced frequency maps.

The framework of the code was designed to allow straightforward additions of any other beam-like observables as in Waelkens et al. (2008), and Sun et al. (2008).

\section{Conclusion}

We have presented the HAMMURABI code, a tool for simulating rotation-measure maps, total and polarized Galactic synchrotron-emission maps taking into account Faraday rotational-depolarization effects, and UHECR deflection maps.

By considering a set of well-known input models from the literature (NE2001 for the thermal electron distribution, Sun et al. 2008; and Page et al. 2007 for the cosmic-ray electron distribution and the Galactic magnetic field), we provided example outputs, comparing them to corresponding observations to illustrate the code's abilities as a scientific tool for charting the Galactic magnetized plasma and the cosmic-ray electron distribution. Full galaxy simulations are currently limited by the finite HEALPix-grid resolution, which prohibit us from probing fluctuations with characteristic lengths of less than $4 \mathrm{pc}$. A second caveat is that the widely used approximation of a power-law energy distribution of the cosmic-ray electrons has been shown to be questionable by sophisticated cosmic-ray propagation simulations (Strong et al. 2007). The degree to which this affects the precision of our simulations has yet to be assessed. Furthermore, our non-ray-tracing calculations of UHECR deflections may not fulfill the strict precision requirements when the UHECR's trajectory through the Galaxy becomes deflected sufficiently, but serve rather as a first approximation.

It was shown that, unsurprisingly, models designed to fit only a fraction of the available observational data of GMF might fail to reproduce the remaining observational information not taken into account in their construction. This is an indication that constraints on the GMF might be highly degenerate. Hence, confronting the models with the broadest possible range of observations of the Galactic magnetic field is paramount to achieving any reliable statements about the field. The motivation for multiobservation constraints, as completed by Sun et al. (2008), was

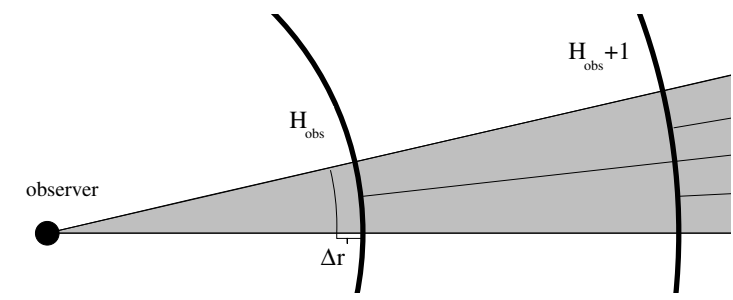

Fig. A.1. An example of the 3D HEALPix grid. For simplicity, it is presented in $2 \mathrm{D}$. The gray shaded area corresponds to the integration volume.

well illustrated by Gaensler et al. (2008), who confirms the idea of a thermal electron density scale height twice as high as that used by the NE2001 model.

Furthermore we have illustrated HAMMURABI's capabilities in generating low-frequency mock observations, where Faraday effects play a significant role. This can be used in feasibility studies and the analysis of true observations by forthcoming lowfrequency radio telescopes such as LOFAR or SKA (note that the latter can also observe at high-frequencies).

HAMMURABI can be applied in constraining the Galactic magnetized plasma and increasing our understanding of radio Galactic emission and UHECR source locations. Already a worthy scientific goal by itself, it will also have implications of our understanding on foreground emission subtraction in experiments such as Planck or WMAP.

Acknowledgements. AHW thanks Xiaohui Sun for remarkably good bug hunting and fruitful discussions; Ronnie Jansson for useful comments and discussions in the development of the code; Wolfgang Reich for fruitful discussions on radio astronomy; Andrew Strong for enlightening discussions about the cosmicray electron population properties; Tony Banday for useful comments on the manuscript; Christoph Pfrommer for corrections to the manuscript; Sam Leach for spotting a bug on the free-free computations; Peter Coles for providing the RM data files; James Cordes and Joseph Lazio for kindly allowing us to make an altered version of their NE2001 software available on the internet and the annonymous referee for useful comments on the manuscript. Some of the results in this paper have been derived using the HEALPix (Górski et al. 2005) package. We acknowledge the use of the Legacy Archive for Microwave Background Data Analysis (LAMBDA). Support for LAMBDA is provided by the NASA Office of Space Science.

\section{Appendix A: The 3D HEALPix grid}

The HAMMURABI code generates HEALPix maps with an angular resolution defined by the parameter NSIDE $=2^{k}$, where $k \in[0,13]$ (the maximum $k$ is a computational limit; it could be extended only by alterations to the original HEALPix package). The integration volume has a cone-like geometry. To minimize the non-homogeneous volume sampling induced by that, the integration volume is consecutively subdivided into so-called subbeams. Each section of these subbeams is contained in a shell centered on Earth. We shall call this grid the 3D HEALPix grid.

This subdivision depends on

- the radial length $\Delta r$ of each volume unit in the observation beam;

- how many shells $H_{\max }$ in total there will be;

- what resolution, or equivalently what NSIDE $_{\text {obs }}$ the observation shell has;

- and finally the shell number of the observation shell $H_{\text {obs }}$ (See an example in Fig. A.1). 
The distances at which the beam is split, or equivalently the upper boundaries ${ }^{4}$ of the shells, are given by

$d_{i}=R_{\max } 2^{i-H_{\max }}$,

where the index $i \in\left[1, H_{\max }\right]$. Due to the disk-like shape of our galaxy, the effective radius out to which we perform our computations varies and decreases sharply towards high latitudes and towards the anti-center direction.

The maximum volume for each shell is a constant corresponding approximately to

$V_{\max }=4 \pi d_{H_{\mathrm{obs}}}^{2} \frac{\Delta r}{12 \cdot \mathrm{NSIDE}_{\mathrm{obs}}^{2}}$.

We suggest ${ }^{5}$ a definition of $\Delta r$ such that the maximum volume unit at the largest shell is close to a cubic form.

We note that the maximum volume unit $V_{\max }$ decreases with higher observation resolution. It defines the minimum scale that can be resolved wit the code for a certain resolution. Fluctuations smaller than that cannot be resolved by the code. This is a formal upper limit, since the Galactic regions more likely to contain small-scale fluctuations are probably not at the far end of the observation volume, where $V_{\max }$ is located, but instead at regions closer to the observer, where the spatial resolution is finer.

\section{A.1. Line of sight integration}

For the $i$ th volume element of a cone, according to the formulae in Sect. 3, we compute the following,

$$
\left\{\begin{aligned}
I_{i}^{\mathrm{syn}} & =C_{I} B_{i, t}^{(1+p) / 2} v^{(1-p) / 2} \mathrm{e}^{-\tau_{i}} \Delta r \\
P_{i} & =C_{\mathrm{P}} B_{i, \perp}^{(1+p) / 2} v^{(1-p) / 2} \mathrm{e}^{-\tau_{i}} \Delta r \\
\Delta R M_{i} & =\frac{a_{0}}{2}\left(n_{\mathrm{e}, i-1} B_{\mathrm{LOS}, i-1}+n_{\mathrm{e}, i} B_{\mathrm{LOS}, i}\right) \Delta r \\
\chi_{i} & =\sum_{j=1}^{i} \mathrm{RM}_{j} \lambda^{2}+\chi_{i, 0} \\
Q_{i} & =P_{i} \cos \left(2 \chi_{i}\right) \\
U_{i} & =P_{i} \sin \left(2 \chi_{i}\right) \\
B_{x, i} & =B_{i, t} \cos \left(\chi_{i, 0}\right) \\
B_{y, i} & =B_{i, t} \sin \left(\chi_{i, 0}\right) \\
\Delta \tau_{i} & =\frac{8.235}{2} \times 10^{-2} v^{-2.1}\left(T_{\mathrm{e}, i-1}^{-1.35} E M_{i-1}+T_{\mathrm{e}, i}^{-1.35} E M_{i}\right) \\
\Delta E M_{i} & =n_{\mathrm{e} i}^{2} \Delta r \\
I_{i}^{f f} & =0.5\left(T_{\mathrm{e}, i-1}+T_{\mathrm{e}, i}\right) \mathrm{e}^{-\tau_{i}}\left(\mathrm{e}^{\Delta \tau_{i}}-1\right) .
\end{aligned}\right.
$$

We note that the calculated Stokes values $U_{i}$ and $Q_{i}$ for each volume element include the effect of foreground RM. Furthermore, total and polarized synchrotron emission are subject to free-free absorption (the $\mathrm{e}^{-\tau}$ term), which is only relevant at frequencies well below $408 \mathrm{MHz}$ (see e.g. Sun et al. 2008). The CR electrons are assumed to follow a power law with an energy spectral index $p$ (see Sect. 3.2). The values $C_{I}$ and $C_{P}$ are dependent on the spatially dependent spectral index $p$ and the spatial distribution component of the cosmic rays $C$, and can be obtained from formulae given in Sect. 3 and Rybicki \& Lightman (1979). The intrinsic polarization angle $\chi_{i, 0}$ is locally defined as zero if the magnetic field is pointing in a positive Galactic-coordinates $l$-direction and $\pi / 2$ when the magnetic field is pointing in a positive Galactic-coordinates $b$-direction. The free-free emission is

\footnotetext{
${ }_{4}$ The code will automatically round down $d_{i}$ to a multiple of $\Delta r$.

${ }^{5}$ Choices of any other constant are of course possible.
}

taken from Rohlfs \& Wilson (1996). The spatially varying thermal electron temperature $T_{\mathrm{e}}$ is given by Eq. (11). Finally, depending on the user's needs, not all computations in Eq. (A.3) are necessary and can be deactivated.

The intensities, RM, $\tau$, and UDMs for a pixel are straightforward to compute. They consist of an integral of the contributions from all volume units below (Eq. (A.4)). For the intensities, in case of a subdivision of the observation cone into subcones, the computation is completed by averaging the set of subcones. However, this is not the case for the UDM, $\tau$, and RM values, since they are found to be linearly dependent on $\lambda^{3}, \lambda^{2.1}$ and $\lambda^{2}$ respectively, where $\lambda$ is the wavelength. Beam averaging would destroy this linear behavior ${ }^{6}$. The UHECR deflection measure is given by $U D M=\sqrt{U D M_{x}^{2}+U D M_{y}^{2}}$, while the deflection orientation is given by $\Theta_{\mathrm{defl}}=\arctan \frac{U D M_{x}}{U D M_{y}}$.

$$
\left\{\begin{array}{cl}
I & =\sum_{i} I_{i} \\
Q & =\sum_{i} Q_{i} \\
U & =\sum_{i} U_{i} \\
R M_{i} & =\sum_{j}^{i} \Delta R M_{j} \\
\tau_{i} & =\sum_{j}^{i} \Delta \tau_{j} \\
U D M_{x} & =\sum_{i} B_{x, i} \Delta r \\
U D M_{y} & =\sum_{i} B_{x, i} \Delta r .
\end{array}\right.
$$

\section{Appendix B: Magnetic field models}

For the convenience of the reader we present the parameterizations of the Galactic magnetic field following Page et al. (2007) and Sun et al. (2008). All parameters are presented in the usual cylindrical coordinates, with respect to an origin located at the Galactic center. The $x$-axis of the coordinate system points in the opposite direction of the Sun, while the $z$-axis points towards the Galactic north.

- Page et al. (2007) writes

$$
\begin{aligned}
\boldsymbol{B}(r, \phi, z)= & B_{0}[\cos \psi(r) \cos \chi(z) \hat{r} \\
& +\sin \psi(r) \cos \chi(z) \hat{\phi}+\sin \chi(z) \hat{z}] .
\end{aligned}
$$

Here $\psi(r)=\psi_{0}+\psi_{1} \ln (r / 8 \mathrm{kpc}), \chi(z)=\chi_{0} \tanh (z / 1 \mathrm{kpc})$. The radial variable $r \in[3 \mathrm{kpc}, 8 \mathrm{kpc}], \chi_{0}=25^{\circ}, \psi_{1}=0.9^{\circ}$ and $\psi_{0}=27^{\circ} . B_{0}$ is not specified in Page et al. (2007) and we put $B_{0}=4 \mu \mathrm{G}$.

- While Sun et al. (2008) presents some suggestions for fields, and we select their $A S S+R I N G$ parameterization:

$$
\left\{\begin{array}{l}
B_{\hat{r}}^{D}=D_{1}(R, \phi, z) D_{2}(R, \phi, z) \sin p \\
B_{\hat{\phi}}^{D}=-D_{1}(R, \phi, z) D_{2}(R, \phi, z) \cos p \\
B_{\hat{z}}^{D}=0
\end{array}\right.
$$

where

$$
D_{1}(r, z)=\left\{\begin{array}{cc}
B_{0} \exp \left(-\frac{r-R_{\odot}}{R_{0}}-\frac{|z|}{z_{0}}\right) & r>R_{c} \\
B_{c} & r \leq R_{c}
\end{array}\right.
$$

6 The final RM, $\tau$, and UDM value is defined as the sum of the highest possible resolution sub-beam of all parent beam contributions. Thus, one intensity pixel might have several corresponding RM, $\tau$, and UDM pixels. 
and $R_{0}=10 \mathrm{kpc}, z_{0}=1 \mathrm{kpc}, R_{c}=5 \mathrm{kpc}, B_{0}=2 \mu \mathrm{G}$, and $B_{c}=2 \mu \mathrm{G}$. and

$D_{2}(r)= \begin{cases}+1 & r>7.5 \mathrm{kpc} \\ -1 & 6 \mathrm{kpc}<r \leq 7.5 \mathrm{kpc} \\ +1 & 5 \mathrm{kpc}<r \leq 6 \mathrm{kpc} \\ -1 & r \leq 5 \mathrm{kpc}\end{cases}$

while the halo field is given by,

$$
B_{\phi}^{H}(r, z)=B_{0}^{H} \frac{1}{1+\left(\frac{|z|-z_{0}^{H}}{z_{1}^{H}}\right)^{2}} \frac{r}{R_{0}^{H}} \exp \left(-\frac{r-R_{0}^{H}}{R_{0}^{H}}\right)
$$

and the parameters are $z_{0}^{H}=1.5 \mathrm{kpc}, B_{0}^{H}=10 \mu \mathrm{G}, R_{0}^{H}=$ $4 \mathrm{kpc}, z_{1}^{H}=0.2 \mathrm{kpc}$ (for $|z|<z_{0}^{H}$ ), and $z_{1}^{H}=0.4 \mathrm{kpc}$ (otherwise).

\section{References}

Beck, R. 2001, Space Sci. Rev., 99, 243

Beck, R., Brandenburg, A., Moss, D., Shukurov, A., \& Sokoloff, D. 1996, ARA\&A, 34, 155

Berkhuijsen, E. M., Mitra, D., \& Mueller, P. 2006, Astron. Nachr., 327, 82

Beuermann, K., Kanbach, G., \& Berkhuijsen, E. M. 1985, A\&A, 153, 17

Bottino, M., Banday, A. J., \& Maino, D. 2008, MNRAS, 935

Brandenburg, A., \& Subramanian, K. 2005, Phys. Rep., 417, 1

Brentjens, M. A., \& de Bruyn, A. G. 2005, A\&A, 441, 1217

Brown, J. C., Taylor, A. R., Wielebinski, R., \& Mueller, P. 2003, ApJ, 592, L29

Burn, B. J. 1966, MNRAS, 133, 67

Clarke, T. E., \& Ensslin, T. A. 2006, AJ, 131, 2900

Cordes, J. M., \& Lazio, T. J. W. 2002 [arXiv: astro-ph/0207156]

Cordes, J. M., \& Lazio, T. J. W. 2003, [arXiv: astro-ph/0301598]

de Oliveira-Costa, A., Tegmark, M., Gaensler, B. M., et al. 2008, MNRAS, 388, 247

Dineen, P., \& Coles, P. 2005, MNRAS, 362, 403

Drury, L. O. 1983, Reports of Progress in Physics, 46, 973

Dunkley, J., Komatsu, E., Nolta, M. R., et al. 2008 [arXiv: 0811.4280]

Frick, P., Stepanov, R., Shukurov, A., \& Sokoloff, D. 2001, MNRAS, 325, 649

Gaensler, B. M., Dickey, J. M., McClure-Griffiths, N. M., et al. 2001, ApJ, 549, 959

Gaensler, B. M., Madsen, G. J., Chatterjee, S., \& Mao, S. A. 2008 [arXiv: 0808.2550]

Gaisser, T. K., \& Stanev, T. 2004, Phys. Lett., B, 592, 228

Górski, K. M., Hivon, E., Banday, A. J., et al. 2005, ApJ, 622, 759

Govoni, F., Murgia, M., Feretti, L., et al. 2006, A\&A, 460, 425

Guidetti, D., Murgia, M., Govoni, F., et al. 2008, A\&A, 483, 699

Han, J. L., Ferriere, K., \& Manchester, R. N. 2004, ApJ, 610, 820

Han, J. L., Manchester, R. N., Lyne, A. G., Qiao, G. J., \& van Straten, W. 2006, ApJ, 642, 868

Harari, D., Mollerach, S., \& Roulet, E. 2000, J. High Energy Phys., 2, 35

Haslam, C. G. T., Klein, U., Salter, C. J., et al. 1981, A\&A, 100, 209

Haslam, C. G. T., Salter, C. J., Stoffel, H., \& Wilson, W. E. 1982, A\&AS, 47, 1
Haverkorn, M., Katgert, P., \& de Bruyn, A. G. 2003, A\&A, 403, 1045 Haverkorn, M., Gaensler, B. M., Brown, J. C., et al. 2006, ApJ, 637, L33 Haverkorn, M., Brown, J. C., Gaensler, B. M., \& McClure-Griffiths, N. M. 2008, ApJ, 680, 362

Heiles, C. 2000, AJ, 119, 923

Hinshaw, G., Weiland, J. L., Hill, R. S., et al. 2008 [arXiv: 0803.0732]

Jaegers, W. J. 1987, A\&AS, 71, 603

Jansson, R., Farrar, G. R., Waelkens, A., \& Enßlin, T. A. 2007 [arXiv: 0708.2714]

Jelić, V., Zaroubi, S., Labropoulos, P., et al. 2008, MNRAS, 891

Johnston-Hollitt, M., Hollitt, C. P., \& Ekers, R. D. 2004, in The Magnetized Interstellar Medium, 13

Kachelrieß, M., Serpico, P. D., \& Teshima, M. 2007, Astroparticle Physics, 26, 378

Kitaura, F. S., \& Enßlin, T. A. 2008, MNRAS, 389, 497

Miville-Deschênes, M.-A., Ysard, N., Lavabre, A., et al. 2008, A\&A, 490, 1093

Moss, D., \& Sokoloff, D. 2008, A\&A, 487, 197

Page, L., Hinshaw, G., Komatsu, E., et al. 2007, ApJS, 170, 335

Peterson, J. D., \& Webber, W. R. 2002, ApJ, 575, 217

Pohl, M., \& Esposito, J. A. 1998, ApJ, 507, 327

Prouza, M., \& Śmída, R. 2003, A\&A, 410, 1

Quireza, C., Rood, R. T., Bania, T. M., Balser, D. S., \& Maciel, W. J. 2006, ApJ, 653, 1226

Reich, P., \& Reich, W. 1986, A\&AS, 63, 205

Reich, P., \& Reich, W. 1988a, A\&AS, 74, 7

Reich, P., \& Reich, W. 1988b, A\&A, 196, 211

Reynolds, R. J., Haffner, L. M., \& Tufte, S. L. 1999, ApJ, 525, L21

Robertson, H. P. 1940, Proc. Camb. Philos. Soc., 36

Roger, R. S., Costain, C. H., Landecker, T. L., \& Swerdlyk, C. M. 1999, A\&AS, 137,7

Rohlfs, K., \& Wilson, T. L. 1996, Tools of Radio Astronomy, XVI (Berlin, Heidelberg, New York: Springer-Verlag), also Astronomy and Astrophysics Library

Rybicki, G. B., \& Lightman, A. P. 1979, Radiative processes in astrophysics (New York: Wiley-Interscience)

Schekochihin, A. A., \& Cowley, S. C. 2006, Physics of Plasmas, 13, 056501

Schekochihin, A. A., Cowley, S. C., Taylor, S. F., Maron, J. L., \& McWilliams, J. C. 2004, ApJ, 612, 276

Schnitzeler, D. H. F. M., Katgert, P., \& de Bruyn, A. G. 2007, A\&A, 471, L21

Simard-Normandin, M., Kronberg, P. P., \& Button, S. 1981, ApJS, 45, 97

Sokoloff, D., \& Shukurov, A. 1990, Nature, 347, 51

Strong, A. W., Moskalenko, I. V., \& Reimer, O. 2004, ApJ, 613, 962

Strong, A. W., Moskalenko, I. V., \& Ptuskin, V. S. 2007, ARA\&A, 57, 285

Sun, X. H., Reich, W., Waelkens, A., \& Enßlin, T. A. 2008, A\&A, 477, 573

Sur, S., Shukurov, A., \& Subramanian, K. 2007, MNRAS, 377, 874

Taylor, A. R., Gibson, S. J., Peracaula, M., et al. 2003, AJ, 125, 3145

Tegmark, M., \& Efstathiou, G. 1996, MNRAS, 281, 1297

The Pierre Auger Collaboration, Abraham, J., Abreu, P., et al. 2007, Science, 318,938

Tinyakov, P. G., \& Tkachev, I. I. 2002, Astropart. Phys., 18, 165

Tucci, M., Martínez-González, E., Vielva, P., \& Delabrouille, J. 2005, MNRAS, 360,935

Vallée, J. P. 2008, ApJ, 681, 303

Vogt, C., \& Enßlin, T. A. 2005, A\&A, 434, 67

Waelkens, A., Maturi, M., \& Enßlin, T. 2008, MNRAS, 383, 1425

Westfold, K. C. 1959, ApJ, 130, 241

Yan, H., \& Lazarian, A. 2006, ApJ, 653, 1292

Yan, H., \& Lazarian, A. 2008, ApJ, 673, 942 